\title{
Patient Radiation Exposure from Embolo-sclerotherapy of Peripheral Vascular
}

\section{Malformations}

Leeying Giet ${ }^{1}$, Nicholas Evans ${ }^{1}$, Anthie Papadopoulou ${ }^{1,2}$, Mohamed Khalifa ${ }^{1,2}$, Janice Tsui ${ }^{1,}$

${ }^{3,4}$, George Hamilton ${ }^{1,3}$, Jocelyn Brookes ${ }^{1,2}$, Chung Sim Lim ${ }^{1,3,4}$

1. Royal Free Vascular Malformation Service, Department of Vascular Surgery, Royal

Free London NHS Foundation Trust, London, United Kingdom, London

2. Department of Interventional Radiology, Royal Free London NHS Foundation Trust, London, UK

3. Department of Surgical Biotechnology, Division of Surgery \& Interventional Science, Faculty of Medical Sciences, University College London, UK

4. NIHR UCLH Biomedical Research Centres, London, UK

Short title: Radiation Exposure from Embolo-sclerotherapy of Vascular Malformations

\section{Corresponding author}

Mr Chung Sim Lim

Department of Vascular Surgery, Royal Free Hospital, Pond Street, London NW3 2QG,

United Kingdom

Tel no: +44 (0) 207794 0500; Fax No: +44 (0) 2074726278

E-mail: chunglim@nhs.net 


\section{Key words}

Vascular malformation, arteriovenous malformation, embolo-sclerotherapy, sclerotherapy, embolization, ionizing radiation

\section{Table of Contents Summary}

This retrospective study analyzed the radiation exposure for patients who underwent embolosclerotherapy (EST) for peripheral vascular malformations (VMs). The single and cumulative radiation exposure in patients with VMs who had EST were generally less than many common endovascular arterial and deep venous interventions. However, since some patients with VMs received relatively high radiation doses from EST and some require multiple interventions, the authors recommend that clinicians who perform these procedures keep the radiation exposure to a minimum.

\section{Article highlights}

Type of research

Single-center, retrospective, cohort study

Key findings

The median (range) dose-area product, DAP measured for single and cumulative embolosclerotherapy (EST) for peripheral vascular malformation (VM) was $1.26 \mathrm{Gycm}^{2}$ (range 0.00 $-698.36 \mathrm{Gycm}^{2}$ ) and 1.91 $\mathrm{Gycm}^{2}$ (range $0.00-1300.24 \mathrm{Gycm}^{2}$ ), respectively. Significantly higher DAP was found in single and cumulative EST for patients with high-flow VMs than those with low-flow VMs.

Take home message

Though overall patient radiation exposure in EST for patients with VM was relatively low, in comparison with many common endovascular arterial and deep venous interventions, the 
DAP and fluoroscopy time measured in some of these procedures were still high. Therefore, it is important that clinicians who perform these procedures keep the radiation exposure to a minimum.

\section{Abstract \\ Objectives}

Embolo-sclerotherapy (EST) is the mainstay therapy for peripheral vascular malformations (VMs) which involves the exposure of patients to ionising radiation. We aimed to analyze the radiation exposure to patients from EST of peripheral VMs over five years in a single specialist center.

\section{Methods}

All patients who had EST performed at single specialist tertiary centre for peripheral VMs between January $1^{\text {st }} 2013$ and January $8^{\text {th }} 2018$ were identified from a prospectively collected database. Data collection included basic demographics, date of the procedure, anatomical site, flow classification of VMs and procedural details. Radiation exposure, measured in dose-area product (DAP) and fluoroscopy time, of all patients identified to have EST of VM during the period were retrospectively reviewed. Statistical analysis was performed using Mann-Whitney $U$ and Kruskal-Wallis tests for comparison between subgroups. $\mathrm{P}<0.05$ was considered significant.

\section{Results}

A total of 237 patients (median age 30 years; range $1-73$ years) underwent 419 ESTs during the study period. Of these, $61(25.7 \%)$ patients had high-flow VM (HFVM) and underwent $140(33.4 \%)$ ESTs. Meanwhile, $176(74.3 \%)$ patients had low-flow VM (LFVM) and underwent 279 ESTs $(66.6 \%)$. Patients with HFVM had a median of 2 procedures (range 1 13), compared to a median of 1 (range 1 - 6) for LFVM within the study period. The median 
DAP for single and cumulative EST for VM were $1.26 \mathrm{Gycm}^{2}$ (range $0.00-698.36 \mathrm{Gycm}^{2}$ ) and $1.91 \mathrm{Gycm}^{2}$ (range $0.00-1300.24 \mathrm{Gycm}^{2}$ ), respectively. Whereas, the median fluoroscopy time for single and cumulative EST were 19 seconds (range 1 - 3845 seconds) and 30 seconds (range $1-5843$ seconds), respectively. Significantly higher patient radiation exposure in DAP and fluoroscopy time was measured for single and cumulative EST for HFVM when compared with LFVM (both $\mathrm{P}<0.01$; Mann Whitney $U$ ). A significant difference in DAP but not fluoroscopic time were found when anatomical areas of VMs by were compared.

\section{Conclusions}

Patient radiation exposure for EST of peripheral VMs measured in DAP and FT appeared to be generally less than those reported for endovascular arterial and deep venous interventions in the literature. However, some patients with peripheral VMs received relatively high radiation doses by our measures. Therefore, further studies should investigate the long-term side-effects of radiation exposure in these patients and strategies to reduce it. 


\section{Introduction}

Vascular malformations (VMs) are a common, heterogenous group of abnormally developed blood vessels that can be divided into high-flow (HFVM) arteriovenous malformations and low-flow (LFVM) venous, lymphatic and capillary malformations depending on the aetiology involved. The mainstay of treatment for peripheral VMs involves embolo-sclerotherapy (EST), for which fluoroscopy and, therefore, ionizing radiation, is required in many cases ${ }^{1,2}$. The deleterious effects of ionizing radiation are well documented and include an increased risk of malignancy ${ }^{3,4}$. As a result, patient and operator exposure during common endovascular procedures, such as endovascular aortic aneurysm repair (EVAR) and peripheral arterial and venous interventions, including angioplasty and stenting, have already been the subject of investigation ${ }^{5-8}$. The cohort of patients who undergo endovascular treatment for VMs are, in general, more likely to be younger than those treated for arterial disease. In addition to this, multiple procedures may be required during the course of their treatment ${ }^{1,2}$. Despite these inherent concerns, there is currently no published data with regards to the cumulative radiation exposure received by patients undergoing EST for peripheral VMs. This study aimed to determine the radiation exposure received by patients with peripheral VMs undergoing EST requiring fluoroscopy over five years at a single specialist tertiary center.

\section{Methods:}

This is a retrospective audit study of a prospectively collected departmental database, with no patient identifiable data. Hence, institutional review board approval or informed consent was not required.

\section{Patients}


All patients who had EST performed at a single specialist tertiary center for peripheral VMs between January $1^{\text {st }} 2013$ and January $8^{\text {th }} 2018$ were identified from a prospectively collected database. All patients with peripheral VMs treated in our hospital underwent a multidisciplinary team review, which included vascular surgeons, interventional radiologists and a clinical nurse specialist. This directed intervention, including EST. In our practice, only patients with symptomatic and/or rapidly growing peripheral VMs and/or those at risk of causing systemic complication were considered for EST. Patients were treated on joint lists, with a vascular surgeon and an interventional radiologist with a specialist interest in VMs, present. All ESTs of HFVM were performed under selective catheter angiography and direct injection. LFVM were treated with direct injection only.

\section{Data collection}

Data collection included basic demographics, date of the procedure, anatomical site, flow classification of VMs and procedural details. Radiation exposure was measured in dose-area product (DAP) and fluoroscopy time. DAP refers to the radiation absorbed by irradiated tissue multiplied by the area irradiated, which gives a rough estimation of the risk of stochastic effects 7,9,10. Procedures were cross-referenced with computer records and those who did not require endovascular treatment, with no recorded fluoroscopy data or with radiation exposure data not in DAP form, were excluded from analysis. DAP and fluoroscopy times were collected directly through the radiology software Clinical Radiology Information System Live, CRIS Live.

\section{Operating theatres and $C$-arm}

All ESTs were performed either in a vascular hybrid theatre with a ceiling mounted C-arm, (Siemens Artis Zeego, Siemens Healthcare, Erlangen, Germany) or in a vascular operating theatre with a mobile C-arm (Siemens Cios Alpha VA20, Siemens Healthcare, Erlangen, 
Germany). Default settings of a pulse rate of 3.0 - 7.5 pulses/second for background fluoroscopy and two frames per second for digital subtraction angiography acquisitions were used. The fluoroscopy equipment was controlled by a trained radiographer for each procedure. Ultrasound guidance was also used if needed.

\section{Statistical analysis}

Statistical analysis was performed using the Statistical Package for Social Sciences, version 17 (SPSS, Chicago, IL). Data was reported in medians, ranges and frequencies. DAP was rounded to the nearest second decimal point in this report including those of less than $0.05 \mathrm{Gycm}^{2}$, which were rounded to $0.00 \mathrm{Gycm}^{2}$. Comparisons between groups were performed using the Mann-Whitney $U$ or Kruskal-Wallis test depending on number of comparisons. $\mathrm{P}<0.05$ was considered significant.

\section{Results:}

\section{Patient demography}

A total of 237 patients (91 males and 146 females; median age 30 years; range $1-73$ years) underwent 419 ESTs during the study period. Of these, 61 (25.7\%) patients had HFVMs and underwent 140 (33.4\%) ESTs. Meanwhile, 176 (74.3\%) patients had LFVMs and underwent 279 ESTs $(66.6 \%)$. Table I summarizes the anatomical distributions of VM in the studied patients. Patients with HVFM had a higher number of interventions (median 2; range 1-13) when compared to those with LFVM (median 1; range 1-6) requiring EST during the study period .

\section{Dose-area product}

\section{Single EST}


Overall, the median DAP per EST for VM was $1.26 \mathrm{Gycm}^{2}$ (range $0.00-698.36 \mathrm{Gycm}^{2} ; 419$ ESTs). Higher patient radiation exposure in DAP was measured per EST for HFVM (median $2.83 \mathrm{Gycm}^{2}$ and range $0.00-698.36 \mathrm{Gycm}^{2} ; 140 \mathrm{ESTs}$ ) than LFVM (median $0.92 \mathrm{Gycm}^{2}$ and range $\left.0.00-182.14 \mathrm{Gycm}^{2} ; 279 \mathrm{ESTs}\right)(\mathrm{P}<0.01$; Mann Whitney $U)$. A significant difference in DAP per EST of VMs of different anatomical sites was also found ( $p<0.01$; Kruskal-Wallis) (Table II).

\section{Cumulative EST per patient}

The median cumulative DAP from EST per patient in the study period was $1.91 \mathrm{Gycm}^{2}$ (range $0.00-1300.24 \mathrm{Gycm}^{2} ; 237$ patients). Higher cumulative DAP was measured per patient for HFVM (median $6.95 \mathrm{Gycm}^{2}$ and range $0.00-1300.24 \mathrm{Gycm}^{2} ; 61$ patients) when compared with LFVM (median $1.53 \mathrm{Gycm}^{2}$ and range $0.00-182.14 \mathrm{Gycm}^{2} ; 176$ patients) $(\mathrm{P}<0.01 ;$ Mann Whitney $U$ ). A significant difference in cumulative DAP per patient of different anatomical sites was also found in the study period ( $\mathrm{p}<0.01$; Kruskal-Wallis) (Table III).

\section{Fluoroscopy time}

Due to incomplete records of fluoroscopy time, only 176 VM patients with 292 ESTs during the study period were analyzed.

\section{Single EST}

The median fluoroscopy time per procedure was 19 seconds (range $1-3845$ seconds; 292 ESTs). Significantly higher fluoroscopy time per EST was measured in patients with HFVM (median 109 seconds and range 1 - 3486 seconds; 105 ESTs) when compared with those with LFVM (median 12 seconds and range 1 - 1644 seconds; 187 ESTs) ( $<<0.01$; Mann-Whitney 
$U$ ). There were no significant differences in fluoroscopy times for ESTs in relation to the anatomical distribution of VMs.

\section{Cumulative EST per patient}

The median cumulative fluoroscopy time from EST per patient in the study period was 30 seconds (range $1-5843$ seconds; 176 patients). Higher cumulative fluoroscopy times were measured per patient for HFVM (median 295 seconds and range 1 - 5843 seconds; 45 patients) when compared with LFVM (median 20 seconds and range 1 - 1739 seconds; 131 patients) $(\mathrm{P}<0.01$; Mann Whitney $U$ ). There were no significant differences in cumulative fluoroscopy times per patient in relation to the anatomical distribution of VMs ( $\mathrm{P}=0.82$; Kruskal-Wallis).

\section{Discussion:}

The use of fluoroscopic guided interventions and therefore, exposure to ionizing radiation, is well documented to potentially cause harm to patients and operators $6,9,11,12$. These effects can include, but are not limited to, skin erythema, burns, skin damage, cataracts and malignancies

13. Whereas some malignancies, such as leukemia show an age-related risk, depending on the age at exposure ${ }^{3}$, the likelihood is that the development of adverse effects following ionizing exposure is multifactorial, with higher cumulative exposure presenting an increased risk for both patients and operators $6,11,14,15$.

Ionizing radiation exposure has previously been the subject of investigation for endovascular procedures including EVAR, coronary interventions and peripheral arterial and venous angioplasty and stenting 5-9, 15-19. A systematic review by Monastiriotis and colleagues found that for a regular, infrarenal EVAR, the mean radiation exposure to the patient in DAP was $79.48 \mathrm{Gycm}^{2}\left(\text { range } 4.3-619 \mathrm{Gycm}^{2}\right)^{5}$. Despite a $78 \%$ reduction in the radiation dose received 
by patients during coronary angiography, with or without intervention, over a ten-year period, Faroux and colleagues found the overall mean DAP per procedure in 2016 was $19.94 \mathrm{Gycm}^{2}$ with standard deviation of $24.90 \mathrm{Gycm}^{2}{ }^{16}$. Meanwhile, Majewska and colleagues reviewed a series of peripheral arterial angioplasty and stenting which revealed a wide range of radiation exposure according to the site and complexity of the procedure involved. The highest exposures were found for iliac artery TASC II class C and renal artery procedures (mean DAP of 199 $\mathrm{Gycm}^{2}$ and $148 \mathrm{Gycm}^{2}$, respectively). The lowest exposures, with a mean DAP of $6.3 \mathrm{Gycm}^{2}$, was found in femoral artery TASC II class A interventions ${ }^{6}$. More recently, Lim and colleagues concluded that endovascular deep venous interventions for central venous outflow obstruction measured in DAP and fluoroscopic time seemed to be less than and, at most, comparable to anatomically similar arterial interventions ${ }^{8}$.

Although there is a relatively large body of literature on arterial interventions and, more recently, venous procedures, very little is known regarding the radiation exposure of patients undergoing EST for HFVM and LFVM. This is one of the very few studies, if any, in the literature that directly estimated the radiation exposure of patients having EST for peripheral VM; a mainstay intervention for the condition. As expected, patients undergoing procedures for HFVM received a significantly higher radiation exposure in DAP and fluoroscopy times for both single and cumulative exposures compared to those with LFVM. This reflects the often more challenging treatment of the former compared to the latter. This study has also found that both single and cumulative radiation exposures measured in DAP for procedures involving the torso and head and neck were higher than those required for upper and lower limbs. Understanding the patient radiation exposure in EST of VM will help clinicians and other responsible radiation safety officers in developing strategies to minimize risk of ionizing radiation exposure to both patients and operators. 
In our study, we have demonstrated that most patients with both LFVM and HFVM undergoing EST, received lower doses of radiation measured in DAP and fluoroscopy time when compared to many common endovascular interventions. These include percutaneous coronary interventions, percutaneous transluminal angioplasty, stenting of the lower limb arteries, iliofemoral vein, inferior vena cava and EVAR (Table IV $)^{5,7,8,16}$. However, the ranges of DAP and fluoroscopy time for single and cumulative EST of both HFVM and LFVM were large, ranging from almost zero to $1300.24 \mathrm{Gycm}^{2}$ and 1 to 5843 seconds, respectively. This indicates that some patients with VMs did receive relatively high radiation exposures from their treatment and, for a few, as high as that seen in complex endovascular arterial and deep venous interventions, such as EVAR and vena cava stenting 5,8,9. These patients have relatively complex, extensive and diffuse VMs. Meanwhile, those with relatively superficial, small and localized VMs are exposed to negligible radiation from their straightforward EST. Moreover, just as those who require arterial and venous interventions, patients with peripheral VMs, especially those that are complex, extensive and diffuse, may also require more than one EST, as shown in this study. This need increases their cumulative radiation exposure. The EVAR trial, which compared endovascular and open repair of abdominal aortic aneurysms, reported an increased incidence of malignancy, in patients treated endovascularly after 15 years of follow-up ${ }^{20}$. It is concerning that the patients who undergo EST for peripheral VMs, as shown in this study, are likely to be within a younger age group than those who require arterial interventions. Therefore, some of these patients, particularly those with challenging and extensive peripheral VMs, potentially receive a higher cumulative radiation from multiple ESTs, at a younger age and with an expected longer life-span than their arterial counterparts. 
With the potential for such exposure, not only to patients, but also to the operators, a limitation of exposure as much as possible is of importance ${ }^{11,12,14}$. Basic training for protective equipment and an awareness of X-Ray protocols essential for the safety of theatre and radiology personnel ${ }^{9,14,17}$. Operators should be aware of optimal patient and staff positioning to reduce radiation exposure and scatter ${ }^{5,14}$. The judicious use of appropriate collimation and magnification should be employed and the use of extreme angulations should also be limited 14. Further use of low dose and pulsed fluoroscopy will contribute to an overall radiation reduction ${ }^{12,18}$. Lastly, newer digital technology, and the use of non-ionizing radiation imaging modalities including ultrasonography and fusion imaging have contributed in significant dose reductions for endovascular procedures $11,21,22$.

There are several limitations to this study. Firstly, as a single center retrospective study with a relatively small number of patients, potential biases cannot be ruled out. As with any medical procedure with exposure to radiation, the risks need to be quantified and limited as much as possible, with further research into this area required. Secondly, DAP and fluoroscopy time are exposure radiation indices that only provide theoretical radiation risk estimates, which do not factor in individual variations in susceptibility to radiation damage ${ }^{9-11}$. There are many additional factors that determine the radiation dose of a procedure, including the patient's build, operative field, the use of digital subtraction and procedural complexity. DAP and fluoroscopy times were used as they are easily obtained as they are automatically computed by modern fluoroscopy units ${ }^{7,9-11,23}$. Furthermore, many studies on radiation exposure, including those for endovascular arterial and deep venous interventions, have reported their findings using these indices, allowing for comparison between studies $5,7,8,16$. However, these comparisons between procedures for differing pathologies should be made cautiously, due the differences in the nature of the pathology, complexity of the interventions and in the patient cohorts studied. 


\section{Conclusion:}

Patient radiation exposure for EST of peripheral VMs measured in DAP and FT appeared to be generally lower than those reported for endovascular arterial and deep venous interventions in the literature. However, some patients with peripheral VMs received relatively high radiation doses from EST. Furthermore, patients with peripheral VMs are usually much younger than those with arterial diseases and may require multiple interventions, leading to further radiation exposure during their lifetime. Further studies should investigate the long-term side-effects of radiation exposure in these patients and strategies to reduce this.

\section{References:}

1. Lee BB, Baumgartner I, Berlien HP, Bianchini G, Burrows P, Do YS, Ivancev K, Kool LS, Laredo J, Loose DA, Lopez-Gutierrez JC, Mattassi R, Parsi K, Rimon U, Rosenblatt M, Shortell C, Simkin R, Stillo F, Villavicencio L, Yakes W. Consensus Document of the International Union of Angiology (IUA)-2013. Current concept on the management of arterio-venous management. Int Angiol. 2013;32(1):9-36.

2. Mulligan PR, Prajapati HJ, Martin LG, Patel TH. Vascular anomalies: classification, imaging characteristics and implications for interventional radiology treatment approaches. Br J Radiol. 2014;87(1035):20130392.

3. United Nations Scientific Committee on Atomic Radiation. Sources, Effects and Risks of Ionizing Radiation. Volume II: Effects of Radiation Exposure of Children. New York: United Nations; 2013. https://www.unscear.org/docs/reports/2013/1385418_Report_2013_Annex_A.pdf 
4. Preston DL, Ron E, Tokuoka S, Funamoto S, Nishi N, Soda M, Mabuchi K, Kodama K. Solid cancer incidence in atomic bomb survivors: 1958-1998. Radiat Res. 2007;168(1):1-64.

5. Monastiriotis S, Comito M, Labropoulos N. Radiation exposure in endovascular repair of abdominal and thoracic aortic aneurysms. J Vasc Surg. 2015;62(3):753-761.

6. Majewska N, Blaszak MA, Juszkat R, Frankiewicz M, Makalowski M, Majewski W. Patients' radiation doses during the implantation of stents in carotid, renal, iliac, femoral and popliteal arteries. Eur J Vasc Endovasc Surg. 2011;41(3):372-377.

7. Spira D, Kirchner S, Blumenstock G, Herz K, Ketelsen D, Wiskirchen J, Wiesinger B. Therapeutic angiographic procedures: differences in dose area product between analog image intensifier and digital flat panel detector. Acta Radiol. 2016;57(5):587594.

8. Lim CS, Waseem S, El-Sayed T, Budge J, Quintana B, Thulasidasan N, Karunanithy N, Black SA. Patient radiation exposure for endovascular deep venous interventions. $J$ Vasc Surg Venous Lymphat Disord. 2019.

9. de Ruiter QM, Reitsma JB, Moll FL, van Herwaarden JA. Meta-analysis of Cumulative Radiation Duration and Dose During EVAR Using Mobile, Fixed, or Fixed/3D Fusion C-Arms. J Endovasc Ther. 2016;23(6):944-956.

10. Coles DR, Smail MA, Negus IS, Wilde P, Oberhoff M, Karsch KR, Baumbach A. Comparison of radiation doses from multislice computed tomography coronary angiography and conventional diagnostic angiography. J Am Coll Cardiol. 2006;47(9):1840-1845.

11. El-Sayed T, Patel AS, Cho JS, Kelly JA, Ludwinski FE, Saha P, Lyons OT, Smith A, Modarai B. Radiation Induced DNA Damage in Operators Performing Endovascular Aortic Repair. Circulation. 2017. 
12. Stecker MS, Balter S, Towbin RB, Miller DL, Vano E, Bartal G, Angle JF, Chao CP, Cohen AM, Dixon RG, Gross K, Hartnell GG, Schueler B, Statler JD, de Baere T, Cardella JF. Guidelines for patient radiation dose management. J Vasc Interv Radiol. 2009;20(7 Suppl):S263-273.

13. Little MP. Risks associated with ionizing radiation. Br Med Bull. 2003;68:259-275.

14. Haqqani OP, Agarwal PK, Halin NM, Iafrati MD. Minimizing radiation exposure to the vascular surgeon. J Vasc Surg. 2012;55(3):799-805.

15. Jones C, Badger SA, Boyd CS, Soong CV. The impact of radiation dose exposure during endovascular aneurysm repair on patient safety. J Vasc Surg. 2010;52(2):298302.

16. Faroux L, Blanpain T, Nazeyrollas P, Tassan-Mangina S, Herce B, Tourneux C, Metz D. Trends in Patient Exposure to Radiation in Percutaneous Coronary Interventions Over a 10-Year Period. Am J Cardiol. 2017;120(6):927-930.

17. Hertault A, Maurel B, Midulla M, Bordier C, Desponds L, Saeed Kilani M, Sobocinski J, Haulon S. Editor's Choice - Minimizing Radiation Exposure During Endovascular Procedures: Basic Knowledge, Literature Review, and Reporting Standards. Eur J Vasc Endovasc Surg. 2015;50(1):21-36.

18. Maurel B, Sobocinski J, Perini P, Guillou M, Midulla M, Azzaoui R, Haulon S. Evaluation of radiation during EVAR performed on a mobile C-arm. Eur J Vasc Endovasc Surg. 2012;43(1):16-21.

19. Miller DL, Balter S, Cole PE, Lu HT, Berenstein A, Albert R, Schueler BA, Georgia JD, Noonan PT, Russell EJ, Malisch TW, Vogelzang RL, Geisinger M, Cardella JF, George JS, Miller GL, 3rd, Anderson J. Radiation doses in interventional radiology procedures: the RAD-IR study: part II: skin dose. J Vasc Interv Radiol. 2003;14(8):977-990. 
20. Patel R, Sweeting MJ, Powell JT, Greenhalgh RM. Endovascular versus open repair of abdominal aortic aneurysm in 15-years' follow-up of the UK endovascular aneurysm repair trial 1 (EVAR trial 1): a randomised controlled trial. Lancet. 2016;388(10058):2366-2374.

21. Maurel B, Hertault A, Sobocinski J, Le Roux M, Gonzalez TM, Azzaoui R, Saeed Kilani M, Midulla M, Haulon S. Techniques to reduce radiation and contrast volume during EVAR. J Cardiovasc Surg (Torino). 2014;55(2 Suppl 1):123-131.

22. Ephrem G, Lau JF, Meraj PM. The fluoro-less and contrast-less peripheral endovascular intervention: a concept for the future today. Cardiovasc Revasc Med. 2015;16(5):294-298.

23. Nyheim T, Staxrud LE, Jorgensen JJ, Jensen K, Olerud HM, Sandbaek G. Radiation exposure in patients treated with endovascular aneurysm repair: what is the risk of cancer, and can we justify treating younger patients? Acta Radiol. 2017;58(3):323330. 
Table I. Anatomical distribution of the peripheral vascular malformations of the patients included in this study. VM: vascular malformation

\begin{tabular}{|l|l|l|c|}
\hline \multicolumn{1}{|c|}{ Anatomical site } & Type of VM & \multicolumn{2}{|c|}{ Number of patients } \\
\hline \multirow{2}{*}{ Head and Neck } & High-flow & 13 & 48 \\
& Low-flow & 35 & \\
\hline \multirow{2}{*}{ Upper Limb } & High-flow & 27 & 68 \\
\hline \multirow{2}{*}{ Lower Limb } & Low-flow & 41 & 84 \\
\hline \multirow{2}{*}{ Torso } & High-flow & 13 & \\
& Low-flow & 71 & 37 \\
\cline { 2 - 4 } & High-flow & 8 & $\mathbf{2 3 7}$ \\
\hline
\end{tabular}


Table II. Patient radiation exposure in dose-area product (DAP) per embolo-sclerotherapy for peripheral vascular malformations of different anatomical sites. *Kruskal-Wallis test.

\begin{tabular}{|c|c|c|c|c|}
\hline $\begin{array}{c}\text { Anatomical } \\
\text { site }\end{array}$ & $\begin{array}{c}\text { Number of Embolo- } \\
\text { sclerotherapies }\end{array}$ & $\begin{array}{c}\text { Median DAP } \\
\left(\mathrm{Gycm}^{2}\right)\end{array}$ & $\begin{array}{c}\text { Range DAP } \\
\left(\mathrm{Gycm}^{2}\right)\end{array}$ & P value* \\
\hline Head and neck & 94 & 4.05 & $0.00-246.51$ & \multirow{4}{*}{$<0.01$} \\
\hline Upper limb & 138 & 0.48 & $0.00-698.36$ & \\
\hline Lower limb & 128 & 1.38 & $0.00-60.23$ & \\
\hline Torso & 59 & 7.28 & $0.00-405.40$ & \\
\hline
\end{tabular}


Table III. Cumulative patient radiation exposure in dose-area product (DAP) per patient for peripheral vascular malformations of different anatomical sites. *Kruskal-Wallis test.

\begin{tabular}{|c|c|c|c|c|}
\hline $\begin{array}{c}\text { Anatomical } \\
\text { site }\end{array}$ & Number of patients & $\begin{array}{c}\text { Median DAP } \\
\qquad\left(\mathrm{Gycm}^{2}\right)\end{array}$ & $\begin{array}{c}\text { Range DAP } \\
\left(\mathrm{Gycm}^{2}\right)\end{array}$ & P value* \\
\hline Head and neck & 48 & 5.19 & $0.00-1300.24$ & \\
\hline Upper limb & 68 & 0.90 & $0.00-817.52$ & $<0.01$ \\
\hline Lower limb & 84 & 1.66 & $0.00-73.40$ & \\
\hline Torso & 37 & 13.40 & $0.00-748.02$ & \\
\hline
\end{tabular}


Table IV. Comparative radiation exposures in dose-area product and fluoroscopy time with endovascular procedures in previously published literature.

\begin{tabular}{|c|c|c|}
\hline Interventions & $\begin{array}{l}\text { Median / Mean (range) } \\
\text { DAP, Gycm² }\end{array}$ & $\begin{array}{l}\text { Median / Mean (range) } \\
\text { FT, seconds }\end{array}$ \\
\hline $\begin{array}{l}\text { Embolo-sclerotherapy of } \\
\text { peripheral high-flow vascular } \\
\text { malformations (this study) }\end{array}$ & $\begin{array}{l}\text { Median } 2.8 \\
\text { (Range } 0.0-698.4 \text { ) }\end{array}$ & $\begin{array}{l}\text { Median } 109 \\
\text { (Range } 1-3486 \text { ) }\end{array}$ \\
\hline $\begin{array}{l}\text { Embolo-sclerotherapy of } \\
\text { peripheral low-flow vascular } \\
\text { malformations (this study) }\end{array}$ & $\begin{array}{l}\text { Median } 0.9 \\
\text { (Range } 0.0-182.1 \text { ) }\end{array}$ & $\begin{array}{l}\text { Median } 12 \\
\text { (Range } 1-1644 \text { ) }\end{array}$ \\
\hline $\begin{array}{l}\text { Percutaneous coronary } \\
\text { interventions }^{16}\end{array}$ & $\begin{array}{l}\text { Mean } 19.9 \\
\text { (+/- standard deviation } \\
\text { 24.9) }\end{array}$ & $\begin{array}{l}\text { Mean } 582 \\
(+/- \text { standard deviation } \\
672)\end{array}$ \\
\hline $\begin{array}{l}\text { Percutaneous transluminal } \\
\text { angioplasty and stent lower leg }{ }^{7}\end{array}$ & $\begin{array}{l}\text { Mean } 6.5 \\
\text { (Range } 4.1-10.5)\end{array}$ & Mean 833 \\
\hline $\begin{array}{l}\text { Percutaneous transluminal } \\
\text { angioplasty and stent lower } \\
\text { (pelvis) }^{7}\end{array}$ & $\begin{array}{l}\text { Mean } 66.9 \\
\text { (Range } 50.2-89.1)\end{array}$ & Mean 535 \\
\hline $\begin{array}{l}\text { Endovascular repair of infrarenal } \\
\text { abdominal aortic aneurysm }{ }^{5}\end{array}$ & $\begin{array}{l}\text { Mean } 79.5 \\
\text { (Range } 4.3-619.0)\end{array}$ & - \\
\hline $\begin{array}{l}\text { Unilateral chronic iliofemoral } \\
\text { venous stenting }^{8}\end{array}$ & $\begin{array}{l}\text { Median } 32.4 \\
(0.1-289.6)\end{array}$ & Median $660(246-4200)$ \\
\hline $\begin{array}{l}\text { Endovascular inferior vena caval } \\
\text { reconstruction }^{8}\end{array}$ & Median $60.8(2.5-269.1)$ & $\begin{array}{l}\text { Median } 2846(836- \\
11682)\end{array}$ \\
\hline
\end{tabular}

